\title{
Model for Estimation of Bounds in Digital Coding of Seabed Images
}

\author{
Andreja SAMCOVIC \\ Faculty of Transport and Traffic Engineering, University of Belgrade, 11040 Belgrade, Serbia \\ andrej@sf.bg.ac.rs
}

\begin{abstract}
This paper proposes the novel model for estimation of bounds in digital coding of images. Entropy coding of images is exploited to measure the useful information content of the data. The bit rate achieved by reversible compression using the rate-distortion theory approach takes into account the contribution of the observation noise and the intrinsic information of hypothetical noise-free image. Assuming the Laplacian probability density function of the quantizer input signal, SQNR gains are calculated for image predictive coding system with non-adaptive quantizer for white and correlated noise, respectively. The proposed model is evaluated on seabed images. However, model presented in this paper can be applied to any signal with Laplacian distribution.
\end{abstract}

\section{Keywords}

Image coding, entropy coding, image denoising, rate distortion theory, signal to noise ratio

\section{Introduction}

Information-theoretic image analysis has aim at defining and measuring the quality of digital images [1]. The information content of the image data can be evaluated objectively both from the signal-to-quantizing noise ratio $(S Q N R)$ and from the entropy of digital images [2]. The preliminary information-theoretic approach may suggest which image bands are more significant, i.e. potentially capable of conveying the amount of information larger than other bands, in order to reduce the volume of data to be processed without noticeable penalty.

This paper is based on a model suitable for quantifying the information content of digital images. In consequence, information-theoretic assessment is presently an open problem [3]. Accurate estimate of the entropy of an image can only be obtained by suppression, or at least, large reduction of the correlation which exists in natural images [4]. Entropy, indeed, is a measure of statistical information that presents the uncertainty of the source.

One of the problems when compressing images is the noise or other artifacts resulting from imperfect data acqui- sition methods [3]. Therefore, any observation noise introduced by the imaging sensor will result in increment of the entropy rate, which is accompanied by a decrement of the information content useful in application context, according to Shannon's information theory [5]. Estimation of the noise must be carried out at the beginning in order to quantify its contribution to the entropy rate.

The problem of digital images denoising is widely discussed in the literature [6-8]. By assuming additive noise, independent of the image and spatial stationary, the noise parameters can be estimated on the homogeneous areas of the image. The bit rate invoked by the entropy coder can be utilized to yield the estimate of the true information content of the image source, i.e. of the entropy that the source would have if it were noise-free. For this purpose, our model is devised from the rate-distortion theory describing how the relations between entropy and the variance of uncorrelated non-Gaussian source change when a stationary white Gaussian random process is superimposed [9]. The motivation underlying our model is investigation of entropy of the noise-free source from the estimated parameters of noise.

In order to develop a novel model for informationtheoretic image assessment, we used differential pulse code modulation (DPCM) as a predictive method for image denoising [10]. The main goal in the DPCM is image decorrelation and removing of redundancy. Since the DPCM coder is statistical in nature, the commonly used image distortion criteria are normalized mean-square-error (MSE) and $S Q N R$.

M. Kivanz et al. exploited an approximate minimum $M S E$ estimation procedure on the problem of image coding [11]. The research presented in this paper contains theoretical results of estimation on upper bound on $S Q N R$ in the closed form for image DPCM systems with non-adaptive quantizer, by using the $M S E$ estimation procedure.

B. Aiazzi et al. defined the entropy model of the image source and estimated the information content of the noise-free source from the code rate [12]. They assumed the noise was white and Gaussian. In our paper, predictive image systems with white and correlated noise will be compared - DPCM system without and DPCM system with entropy modeling. The performance metric to be used is $S Q N R$ as an objective measure of quality of the recon- 
structed image. Further increase in the SQNR without increasing the transmission rate is possible if we assume epsilon $(\varepsilon)$-entropy of the bit stream at the quantizer input [13].

Fan and Xia developed a new hidden Markov model and applied it to image denoising by exploiting the local statistics of the signal [8]. Our model will imply local statistics through the analysis of the probability density function (PDF) of the input image signal.

Concerning noise modeling, Jerzman and Kicinsky took into account kernel estimation of the PDF [14]. Their research focused on the estimation of the univariate, unimodal data representative for the normal distribution. With this in mind, we used the Laplacian PDF in development of our model and obtained the corresponding numerical results.

A commonly used source model in many digital applications is Laplacian source model, due to its simplicity and fact that many parameters and characteristics can be found as the closed form relations. Also, for larger number of signal samples, the PDF of input signal is better represented with Laplacian functions. In numerous papers, the quantization of memoryless Laplacian source was analyzed since the PDF of the difference signal for an image waveform follows the Laplacian distribution [3], [14-15]. Our model can be extended to any image with Laplacian distribution, but the evaluation will be carried out on seabed images.

Sonar images are perturbed by a multiplicative noise called speckle, due to the coherent nature of the scattering phenomenon. In [16] an image denoising algorithm for sonar images in the wavelet domain was presented, which tends to reduce the speckle, preserving the structural features of the scene.

Despite the actual proliferation of real sonar images, there are not numerous publications dealing with their denoising. A. Isar et al. [17] presented the particular case of sonar images starting with an overview of speckle removal techniques both in the spatial domain and in the wavelet domain.

Aiming at the information loss, serious noisy and low resolution of sonar image, a sonar image fusion denoising method based on multiple morphological wavelet packets is proposed in [18].

This paper is organized as follows. Section 2 introduces information-theoretic analysis, starting gradually from noise modeling, as well as taking into account source decorrelation by DPCM approach and assumed noise models. In Sec. 3, entropy modeling of memoryless images is described. Section 4 invokes the concept of entropy coding. The development of novel analytical model on upper bounds of corresponding performances will be developed in Sec. 5. In Sec. 6, some simulations on several sets of sonar images are carried out. Section 7 contains the concluding remarks.

\section{Noise Modeling}

This section focuses on noise variance modeling from image data. Unlike coherent or systematic disturbances, which may occur in some kind of data, the noise is assumed to happen due to a fully stochastic process. Let us assume for the noise an additive signal-independent model [15]:

$$
x(i, j)=f(i, j)+n(i, j)
$$

in which $x(i, j)$ is the intensity level at the pixel position $(i, j) ; f(i, j)$ is the signal intensity without noise, and $n(i, j)$ is a zero-mean Gaussian process independent of $f$, stationary at the position $(i, j)$, and spatially auto-correlated. Both $x(i, j)$ and $f(i, j)$ are regarded as non-stationary non-Gaussian (auto-correlated) stochastic processes. Let the corresponding variances of the signal (with and without noise) and noise be $\sigma_{x}^{2}, \sigma_{f}^{2}$ and $\sigma_{n}^{2}$, respectively. Thanks to the independence of signal and noise components and to the spatial stationarity of the latter, the variances of sum random variables from (1) can be calculated as follows:

$$
\sigma_{x}^{2}(i, j)=\sigma_{f}^{2}(i, j)+\sigma_{n}^{2}(i, j)
$$

\subsection{Source Decorrelation by DPCM}

Lossless or reversible signal compression consists of source decorrelation and entropy coding. Differential Pulse Code Modulation (DPCM) is usually employed for reversible data compression [15]. DPCM is widely used in image coding. The main advantage of DPCM is its ability to perform efficient coding with low complexity. For a highly correlated source input, the DPCM coder removes correlation by prediction and codes only the residue. The blockscheme of the DPCM system is shown in Fig. 1, where $e$ depicts prediction error, $\hat{x}$ is the prediction signal, and $q$ represents the quantizing noise.

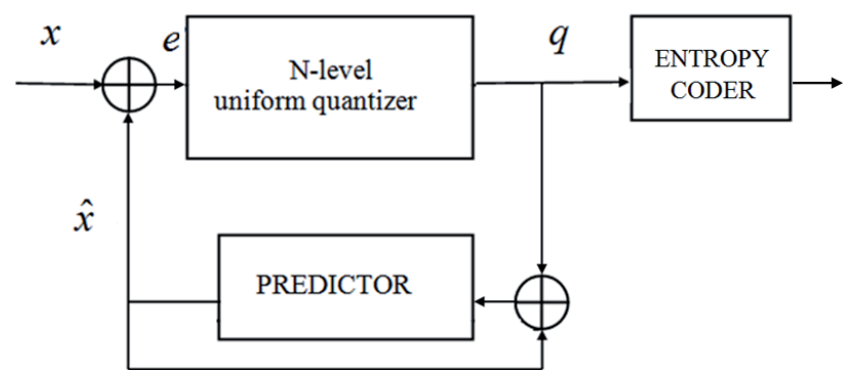

Fig. 1. Blok-scheme of the DPCM system.

DPCM is a technique of converting an analog into a digital signal in which an analog signal is sampled and then the difference between the actual sample value and its predicted value is quantized. A predicted value of the actual sample is based on the value of the previous sample or the values of the previous samples [15]. The concept of DPCM is based on the fact that most source signals show a significant correlation between successive samples so that quantizer uses redundancy in sample values which provides lowering bit rate [15]. 
DPCM basically consists of prediction followed by entropy coding of the resulting prediction errors [19]. The analysis for DPCM algorithm will be developed in this paper. DPCM system with non-adaptive quantizer and entropy coding will be considered. Adaptation of the quantizer can be accomplished by making estimation of the standard deviation before it is quantized. The adaptive quantizers have considerably less idle channel noise than non-adaptive quantizers and are able to operate optimally over a wide range of input signal levels. Unfortunately, adaptation increases the entropy.

For the benefit of clarity, our model will be developed for one-dimensional DPCM system [20]. Prediction will be linear throughout it. Let $\hat{x}(i)$ denotes the prediction at pixel $i$ obtained as a linear regression of the values of $P$ previous pixels [12]:

$$
\hat{x}(i)=\sum_{j=1}^{P} \Phi(j) \cdot x(i-j)
$$

where $\Phi(j)$ represents the coefficients of the linear predictor and is assumed to be constant throughout the image. By replacing the additive noise model from (1), we reached

$$
\hat{x}(i)=\hat{f}(i)+\sum_{j=1}^{P} \Phi(j) \cdot n(i-j)
$$

in which $\hat{f}(i)$ represents the prediction for the noise-free image as formulated from its previous pixels:

$$
\hat{f}(i)=\sum_{j=1}^{P} \Phi(j) \cdot f(i-j) .
$$

Prediction errors $e(i)$ of the input image pixels $x(i)$ are defined by [9]

$$
e(i)=x(i)-\hat{x}(i)=e_{f}(i)+n(i)-\sum_{j=1}^{P} \Phi(j) \cdot n(i-j)
$$

where $e_{f}(i)=f(i)-\hat{f}(i)$ is the error the predictor would produce if it were fed by noise-free data with the corresponding variance factor $\sigma_{e f}^{2}$. Both $e(i)$ and $e_{f}(i)$ are zeromean processes, uncorrelated and non-stationary. The zeromean property stems from an assumption of local firstorder stationarity within the $(P+1)$-pixel window comprising both the current pixel and its prediction support.

\subsection{White Noise Model}

Let us assume that the noise $n(i)$ is white and Gaussian, i.e. a stationary and uncorrelated random process with zero mean and variance $\sigma_{n}{ }^{2}$. Under this assumption, the variance of $e(i)$ from (6) becomes [12]

$$
\begin{aligned}
\sigma_{e}{ }^{2}(i) & =\sigma_{e f}{ }^{2}(i)+\sigma_{n}{ }^{2}(i)+\sum_{j=1}^{P} \Phi^{2}(j) \cdot \sigma_{n}{ }^{2}(i) \\
& =\sigma_{e f}{ }^{2}(i)+\sigma_{n}{ }^{2}(i)\left[1+\sum_{j=1}^{P} \Phi^{2}(j)\right]
\end{aligned}
$$

in which the summation term at right-hand stems from the statistical independence and stationarity of noise samples. Term $G=\sum_{j=1}^{P} \Phi^{2}(j)$ refers to the power gain of the linear filter responsible for the prediction. Equation (7) states that the variance of prediction errors calculated on the noisy image equals that of prediction errors calculated on the noise-free image incremented by the noise variance multiplied by $1+G$. This means that any attempt to predict a white noise process results in an increment of the variance of the outcome prediction errors, unless the predictor has coefficients all identical to zero.

\subsection{Correlated Noise Model}

When the noise is correlated, equation (7) no longer holds, because of the statistical dependence of $n(i)$ from the previous noise samples. Let us assume for the stationary zero-mean Gaussian noise the first-order Markov model [15], uniquely defined by the coefficient correlation $\rho$ and the variance $\sigma_{n}{ }^{2}$ :

$$
n(i)=\rho \cdot n(i-1)+\varepsilon_{n}(i)
$$

where $\varepsilon_{n}(i)$ is a white Gaussian uncorrelated random process having variance $\sigma_{\varepsilon n}{ }^{2}$. From (8) it stems that

$$
\sigma_{\varepsilon n}^{2}=\sigma_{n}^{2} \cdot\left(1-\rho^{2}\right)
$$

In the case of the correlated noise, (6) may be written as

$$
e(i)=e_{f}(i)+n(i)-\hat{n}(i)=e_{f}(i)+e_{n}(i)
$$

in which $e_{n}(i)=n(i)-\hat{n}(i)$ is the error produced when the correlated noise is being predicted. This error is assumed to be zero-mean, stationary and independent of $e_{f}(i)$, since $f$ and $n$ are assumed to be independent of each other. The zero-mean additive signal-independent correlated noise model from (1) is relatively simple and mathematically tractable. Its accuracy has been validated for two-dimensional (2-D) signals [6]. Thus, the relation among the variances of the three types of prediction errors becomes

$$
\sigma_{e}^{2}=\sigma_{e f}^{2}+\sigma_{e n}^{2} \text {. }
$$

By replacing the noise model (8) with (11), the variance $\sigma_{e n}^{2}$ can be calculated as

$$
\begin{array}{r}
\sigma_{e n}^{2}=\sigma_{n}^{2}\left\{1+G-2 \sum_{j=1}^{P} \Phi(j) \cdot \rho^{j}+\sum_{j=1}^{P} \sum_{l=1}^{P} \Phi(j) \Phi(l) \rho^{|j-l|}\right\} \\
j \neq l
\end{array}
$$

When the noise is white, i.e. $\rho=0$, that means the third and fourth terms in (12) are equal to zero and in that case it can be easily noticed that (12) reduces to (7). On the other hand, from the noise model (8) it can be concluded that the variance $\sigma_{e n}^{2}$ is lower bounded by $\sigma_{\varepsilon n}^{2}(9)$, which means $\sigma_{e n}^{2} \geq \sigma_{n}^{2} \cdot\left(1-\rho^{2}\right)$. 
The optimal predictor for the first order Markov model in (8) is $\Phi(1)=\rho$ and $\Phi(j)=0$ for $j=2, \ldots, P$; it yields to

$$
\sigma_{e n}^{2}=\sigma_{n}^{2} \cdot\left(1-\rho^{2}\right)=\sigma_{\varepsilon n}^{2}
$$

for the correlated noise, and it can be easily verified. Thus, the residual variance of the noise after decorrelation may be approximated from the estimated variance of the correlated noise, and from its estimated correlation coefficient. The approximation becomes more accurate as the predictor attains the optimal performance. A better understanding of correlation in noise and target signals would provide an additional input to signal processing algorithms.

\section{Entropy Modeling}

The performance of a quantizer can often be improved by variable-length coding of the sequence of the quantizer outputs, i.e. by entropy coding. Entropy coding is a source encoding technique. This is a variable-length procedure that assigns shorter code words to highly probable symbols and longer code words to less probable symbols. When symbols to be transmitted are independent, it is possible to generate codes such that the average word length of these codes is approximately equal to the entropy of the symbols. To understand efficient coding when using entropy coding, it is necessary to keep the quantizing error fixed and obtain quantization characteristics that will minimize the entropy.

Let us assume a stationary memoryless source uniquely defined by its probability density function (PDF) $p(x)$, having zero-mean and variance $\sigma_{e}{ }^{2}$, and the step size $\Delta$ of the uniform quantizer. Wood has shown that when the number of quantizing levels gets large, the minimum bit rate $R$ needed to encode one of its samples approaches [21]:

$$
R \cong h-\log _{2} \Delta .
$$

This approximation gets closer to the true differential entropy as $\Delta$ tends to one. The differential entropy $h$ of the source signal is defined as [22]

$$
h=-\int_{-\infty}^{\infty} p(x) \log _{2} p(x) d x=\frac{1}{2} \log _{2}\left(c \cdot \sigma_{e}^{2}\right)
$$

with $0<c \leq 2 \pi e$ a positive constant accounting for the shape of the PDF and called as entropy factor, while $e=2.718$ is the base of the natural logarithm. The entropy factor attains its maximum $c=2 \pi e$ for the Gaussian density function. The $p(x)$ is an even function representing the probability density of quantizer input. Such quantizer was originally studied by Panter and Dite [22]. Nitadori [23] and O'Neal [10] found that the quantizer input for nonadaptive DPCM image systems has approximately exponential two-sided Laplacian function, i.e:

$$
p(x)=\frac{1}{\sqrt{2} \sigma_{e}} \exp \left(-\frac{\sqrt{2}|x|}{\sigma_{e}}\right)
$$

If the source is non-stationary, as it may happen for prediction errors, it is still possible to define the differential entropy from the average PDF, i.e. a PDF given by the superposition of many space-varying Gaussian PDFs. Such a PDF may be calculated from the whole set of occurrences, same as if the source were stationary, and usually results to be non-Gaussian. However, in this case, the bit rate given by (14) does not necessarily attain the minimum. By classifying the source into a number of quasi stationary Gaussian subsources and by applying (14) to each of the new sources, a moderately lower rate may be achieved [24].

The distortion criterion to be used will be the squarederror criterion. Panter and Dite have shown that the minimum mean-square quantizing error is given by [22]

$$
\sigma_{q}^{2}=\frac{2}{3 N^{2}}\left[\int_{0}^{v} p^{1 / 3}(x) d x\right]^{3}
$$

where $N=2^{n}$ is the total number of the quantizing levels, and $n$ depicts the number of bits per sample. The major assumption is that the limiting degradation factor is general quantizing noise. When the range of the quantizer $v$ in the (17) becomes large [10], we obtain the approximation for the mean-square error of the quantizing noise by solving the integral from (17)

$$
\sigma_{q}^{2}=\frac{9}{2 N^{2}} \sigma_{e}^{2}
$$

In the DPCM system without entropy coding, the quantizer is designed to minimize the ratio $\sigma_{q}{ }^{2} / \sigma_{e}{ }^{2}$ for the fixed number of the quantizing levels $N$. For the DPCM system with entropy coding, the quantizer is designed to minimize $\sigma_{q}{ }^{2} / \sigma_{e}{ }^{2}$ when the entropy of the quantizer output is fixed. For both DPCM systems, this results in minimizing the ratio $\sigma_{q}{ }^{2} / \sigma_{e}{ }^{2}$ and therefore maximizing the $S Q N R$ for the fixed bit rate in a channel.

When the quantizer input has the Laplacian density, the differential entropy $h$ of (15) becomes [15]

$$
h=\log _{2}\left(\sqrt{2} e \sigma_{e}\right) \text {. }
$$

Bennett [25] has shown that if the signal is evenly distributed between equispaced quantizing levels of step size $\Delta$, the mean-square quantizing error is related to the step size by

$$
\varepsilon_{q}^{2}=\frac{1}{12} \Delta^{2}
$$

Let us assume $e(i)$ are regarded as an uncorrelated source and are uniformly quantized with the step size $\Delta$. This represents a high bit rate approximation. The minimum average bit rate $R$ (in bits per pel), necessary to rever- 
sibly encode an integer-valued sample of the signal $x$, which is equal to the entropy of the quantizer output $H$, may be then approximated as in (14) [3]:

$$
R=H=\frac{1}{2} \log _{2} \frac{e^{2} \overline{\sigma_{e}^{2}}}{6 \varepsilon_{q}^{2}}
$$

where $\overline{\sigma_{e}^{2}}$ is the average variance of the prediction error. By averaging (11) and replacing it with (21), $H$ may be then written as

$$
H=\frac{1}{2} \log _{2}\left[\frac{e^{2}}{6 \varepsilon_{q}^{2}}\left(\overline{\sigma_{e f}^{2}}+\sigma_{e n}^{2}\right)\right]
$$

in which $\overline{\sigma_{e f}^{2}}$ is the average variance of the $e_{f}(i)$. If $\overline{\sigma_{e f}^{2}}=0$, then $H$ from (22) becomes $H_{n}$ :

$$
H_{n}=\frac{1}{2} \log _{2}\left[c \cdot \sigma_{e n}^{2}\right]=\frac{1}{2} \log _{2}\left[2 \pi \cdot e \cdot \sigma_{e n}^{2}\right]
$$

because in this case the entropy factor corresponds to the Gaussian PDF of $e_{n}(i)$. Analogously, if ${\sigma_{e n}}^{2}=0$, then (22) reduces to

$$
H_{f}=\frac{1}{2} \log _{2}\left[\frac{e^{2}}{6 \varepsilon_{q}^{2}} \overline{\sigma_{e f}^{2}}\right]
$$

Thus, the average entropy rate of the noise-free image $f$ in the case of white noise will be given by (7) and (24) as

$$
H_{f}=\frac{1}{2} \log _{2}\left\{\frac{e^{2}}{6 \varepsilon_{q}^{2}}\left[\overline{\sigma_{e}^{2}}-(1+G) \cdot \sigma_{n}^{2}\right]\right\}
$$

whereas in the case of correlated noise, replacing (13) with (24) gives

$$
H_{f}=\frac{1}{2} \log _{2}\left\{\frac{e^{2}}{6 \varepsilon_{q}^{2}}\left[\overline{\sigma_{e}^{2}}-\left(1-\rho^{2}\right) \cdot \sigma_{n}^{2}\right]\right\} .
$$

The average variance $\overline{\sigma_{e}^{2}}$ can be measured during the compression procedure by averaging $\sigma_{e}^{2}$, and this determination is crucial for the accuracy of the estimation of $H_{f}$.

\section{Bound on Entropy Modeling}

Since the variance $\sigma_{e}^{2}$ is the same for DPCM systems with and without entropy coding, from (18) and (21), and assuming the constant bit rate in the channel, we obtain the following from (25) for the white noise:

$$
\frac{9}{2} \frac{\sigma_{e}^{2}}{\sigma_{q}^{2}}=\frac{e^{2}}{6 \varepsilon_{q}^{2}}\left[\sigma_{e}^{2}-(1+G) \cdot \sigma_{n}^{2}\right] .
$$

Taking into account the number of quantizing levels $N$ and the fact that the mean-square error of the quantizing noise $\sigma_{q}{ }^{2}$ and the variance of the noise $\sigma_{n}{ }^{2}$ are equal when the number of quantizing levels is large [10], we can obtain the relation between the mean-square quantizing error $\varepsilon_{q}{ }^{2}$ and the mean-square error of the quantizing noise:

$$
\varepsilon_{q}^{2}=\frac{e^{2}}{27}\left[1-\frac{1+G}{N^{2}}\right] \sigma_{q}^{2} .
$$

The realistic value for the coefficient correlation $\rho$ of the image signal is 0.95 [15], i.e. $\rho^{2}=0.902$. For the optimum predictor, the power gain of the linear filter becomes $G=\rho^{2}=0.902$. Without loosing on generality, if we take, for instance, $n=3$ bits per sample and $N=2^{3}=8$ quantizing levels, we can obtain that in such case

$$
\varepsilon_{q}^{2}=0.266 \sigma_{q}^{2}
$$

The following equation (30) gives the relation between the quantizing noise powers, when two observed systems operate at the same bit rate:

$$
\frac{\sigma_{e}^{2}}{\varepsilon_{q}^{2}}=\frac{27}{e^{2}} \frac{1}{\left[1-\frac{1+G}{N^{2}}\right]} \frac{\sigma_{e}^{2}}{\sigma_{q}^{2}} .
$$

This study leads to the conclusion that when the bit rate is large, the quantizing system using entropy coding can achieve the $S Q N R$ of $10 \log \left\{27 /\left[e^{2}\left(1-(1+G) / N^{2}\right)\right]\right\}$ greater than the system without entropy coding. In the case of $N=8$ quantizing levels, the numerical value of the $S Q N R$ gain will be $5.76 \mathrm{~dB}$ with applied entropy coding.

The similar calculation can be carried out in the case of correlated noise. Taking into account (18) and (21), (26) leads to

$$
\frac{9}{2} \frac{\sigma_{e}^{2}}{\sigma_{q}^{2}}=\frac{e^{2}}{6 \varepsilon_{q}^{2}}\left[\sigma_{e}^{2}-\left(1-\rho^{2}\right) \cdot \sigma_{n}^{2}\right] .
$$

If we suppose $N$ number of quantizing levels, the following relation between the mean-square quantizing error and the mean-square error of the quantizing correlated noise can be obtained:

$$
\varepsilon_{q}^{2}=\frac{e^{2}}{27}\left[1-\frac{1-\rho^{2}}{N^{2}}\right] \sigma_{q}^{2} .
$$

If we take the value of $\rho=0.95$ and $N=8$ quantizing levels, the following relation can be obtained:

$$
\varepsilon_{q}^{2}=0.274 \sigma_{q}^{2}
$$

Equation (34) presents the relation between the quantizing noise powers, assuming the two systems (with and without entropy coding):

$$
\frac{\sigma_{e}^{2}}{\varepsilon_{q}^{2}}=\frac{27}{e^{2}} \frac{1}{\left.1-\frac{1-\rho^{2}}{N^{2}}\right]} \frac{\sigma_{e}^{2}}{\sigma_{q}^{2}} .
$$

To conclude, in the case of correlated noise in image coding, the quantizing system using entropy coding can achieve the $S Q N R$ of $10 \log \left\{27 /\left[e^{2}\left(1-\left(1-\rho^{2}\right) / N^{2}\right)\right]\right\}$ 
greater than the system without entropy coding. For $N=8$ quantizing levels, the corresponding value of the SQNR gain will be $5.64 \mathrm{~dB}$ with entropy coding and correlated noise, which is improvement in comparison with existing models [26].

\section{Upper Bound on SQNR Ratio}

A matter often arising in signal quantizing and processing is the minimum average number of bits per sample which is necessary to achieve certain fidelity. The answer to this question is given by epsilon-entropy ( $\varepsilon$-entropy) [13]. The concept of epsilon-entropy was introduced and developed in order to describe the problem of signal transmission with maximum error constraint. Namely, we desire to compress a source to $R$ bits per pixel where it is required that we must be able to reconstruct the original source sequence with error on each pixel that never exceeds $\varepsilon$. The major result of this subject is that the epsilon-entropy can be expressed as an information-theoretic minimization.

Let us assume that in a digital communication system, the information source generates the random signal $x$ which is encoded with an accuracy $\varepsilon$, where $\varepsilon>0$. The random signal $y$ at the encoder's output and the generated signal $x$ belong to the same space. Their associated probability belongs to the same class of distribution, which is determined by $\varepsilon$. Thus, $\varepsilon$-entropy $H_{\varepsilon}$ is an infinum of the mutual information for two random signals, one of which is given as [13], [19]:

$$
H_{\varepsilon}=\inf I(x ; y)
$$

Assume that a signal $x$ arising from the source is expressed by pixel values as well as the restriction that the mean-square error per image pixel should not exceed $\varepsilon^{2}$. When $x$ is an $M$-dimensional random variable and has a sufficiently smooth density function, the $\varepsilon$-entropy $H_{\varepsilon}$ is given as [19]

$$
H_{\varepsilon}=M \log _{2} \frac{1}{\varepsilon}+h-\frac{M}{2} \log _{2} 2 \pi \varepsilon
$$

where $h$ is the differential entropy of the signal [15], [19]. The behavior of $\varepsilon$-entropy is determined in the first place by the dimension of the variable.

Further increase in the signal-to-quantizing noise ratio without increasing the transmission rate is possible if we assume $\varepsilon$-entropy of the bit stream of the quantizer output. For the Laplacian model probability density, the average $\varepsilon$-entropy of the one-dimensional quantizer output using (36) is

$$
H_{\varepsilon}=\frac{1}{2} \log _{2} \frac{e \overline{\sigma_{e}^{2}}}{\pi \varepsilon_{q}^{2}}
$$

whereas $\varepsilon_{q}^{2}$ is the mean-square quantizing error. The uniform quantization includes the equality $\varepsilon^{2}=\varepsilon_{q}{ }^{2}$. Comparing the white quantizing noise of the system in which we as- sume $\varepsilon$-entropy of the bit stream of the quantizer output and the system without entropy coding [27], we shall verify in the framework of our model that the quantizing system using $\varepsilon$-entropy of the bit stream can give the $S Q N R$ greater than the system without entropy coding:

$$
\frac{9}{2} \cdot \frac{\sigma_{e}^{2}}{\sigma_{q}^{2}}=\frac{e}{\pi \varepsilon_{q}^{2}}\left[\sigma_{e}^{2}-(1+G) \cdot \sigma_{n}^{2}\right] .
$$

Finally, it will lead to

$$
\varepsilon_{q}^{2}=\frac{2 e}{9 \pi}\left[1-\frac{1+G}{N^{2}}\right] \sigma_{q}^{2} .
$$

For $N=8$ quantizing levels,

$$
\varepsilon_{q}^{2}=0.186 \sigma_{q}^{2}
$$

Equation (41) gives the relation between the white quantization noise powers when two systems operate at the same bit rate:

$$
\frac{\sigma_{e}^{2}}{\varepsilon_{q}^{2}}=\frac{9 \pi}{2 e} \frac{1}{\left[1-\frac{1+G}{N^{2}}\right]} \frac{\sigma_{e}^{2}}{\sigma_{q}^{2}} .
$$

An optimum quantizer is one that minimizes the mean-square error subject to the constraint of a given output entropy rate. When the bit rate is large, the quantizing system using $\varepsilon$-entropy of the bit stream can give the $S Q N R$ improvement of $10 \log \left\{9 \pi /\left[2 e\left(1-(1+G) / N^{2}\right)\right]\right\}[\mathrm{dB}]$ greater than the system without entropy coding. For $N=8$ quantizing levels, the upper bound on the $S Q N R$ gain for image DPCM system with non-adaptive quantizer and white noise has resulted in $7.29 \mathrm{~dB}$, which indicates the improvement compared with the existing results [28].

The similar procedure will be applied to the correlated noise:

$$
\frac{9}{2} \cdot \frac{\sigma_{e}^{2}}{\sigma_{q}^{2}}=\frac{e}{\pi \varepsilon_{q}^{2}}\left[\sigma_{e}^{2}-\left(1-\rho^{2}\right) \cdot \sigma_{n}^{2}\right]
$$

leading to the following relation:

$$
\varepsilon_{q}^{2}=\frac{2 e}{9 \pi}\left[1-\frac{1-\rho^{2}}{N^{2}}\right] \sigma_{q}^{2} .
$$

In the case of $N=8$ quantizing levels, this relation has the numerical value:

$$
\varepsilon_{q}^{2}=0.192 \sigma_{q}^{2}
$$

The above equation gives the relation between the correlated quantization noise powers:

$$
\frac{\sigma_{e}^{2}}{\varepsilon_{q}^{2}}=\frac{9 \pi}{2 e} \frac{1}{\left[1-\frac{1-\rho^{2}}{N^{2}}\right]} \frac{\sigma_{e}^{2}}{\sigma_{q}^{2}} .
$$

In the considered case of correlated noise, the quantizing system using $\varepsilon$-entropy of the bit stream can give the 
SQNR improvement of $10 \log \left\{9 \pi /\left[2 e\left(1-\left(1-\rho^{2}\right) / N^{2}\right)\right]\right\}$ $[\mathrm{dB}]$ greater than the system without entropy coding. Taking $N=8$ quantizing levels, the upper bound on the $S Q N R$ gain for image DPCM system with non-adaptive quantizer and correlated noise will be $7.17 \mathrm{~dB}$.

The obtained numerical results for $N=8$ quantizing levels in this paper can be summarized in Tab. 1:

\begin{tabular}{|l|c|}
\hline \multicolumn{1}{|c|}{ Method } & SQNR gain $[\mathrm{dB}]$ \\
\hline Entropy coding with white noise & 5.76 \\
\hline Entropy coding with correlated noise & 5.64 \\
\hline $\begin{array}{l}\text { Upper bound (epsilon-entropy coding) } \\
\text { with white noise }\end{array}$ & 7.29 \\
\hline $\begin{array}{l}\text { Upper bound (epsilon-entropy coding) } \\
\text { with correlated noise }\end{array}$ & 7.17 \\
\hline
\end{tabular}

Tab. 1. Obtained numerical results for SQNR gain for $N=8$ quantizing levels.

\section{Experimental Results}

Our model will be evaluated on sonar side-scan images. Side-scan images are generally mounted on the sides of towfish (sensor), which are pulled behind ships, or on automated underwater vehicles [29]. At either side of the vehicle, the seabed is scanned in a series of strips. These strips are stacked together to form an image of the whole seabed. A typical side-scan sonar image is shown in Fig. 2, where the horizontal axis represents pixels, whereas the vertical axis shows scan lines. In order to evaluate our model on seabed images, we used the set of 511 side-scan images captured by the autonomous vehicle system.

The image shows the seabed to each side of the ship's path, which should been vertical, through the centre of the image. Both sides of the sensor are equipped with an array of sonar transducers which can transmit and detect sound. The array of transmitters is used to beam from the signal, aiming it in a narrow beam. This enables the sonar system to cover the seabed in narrow strips. Each row of pixels in

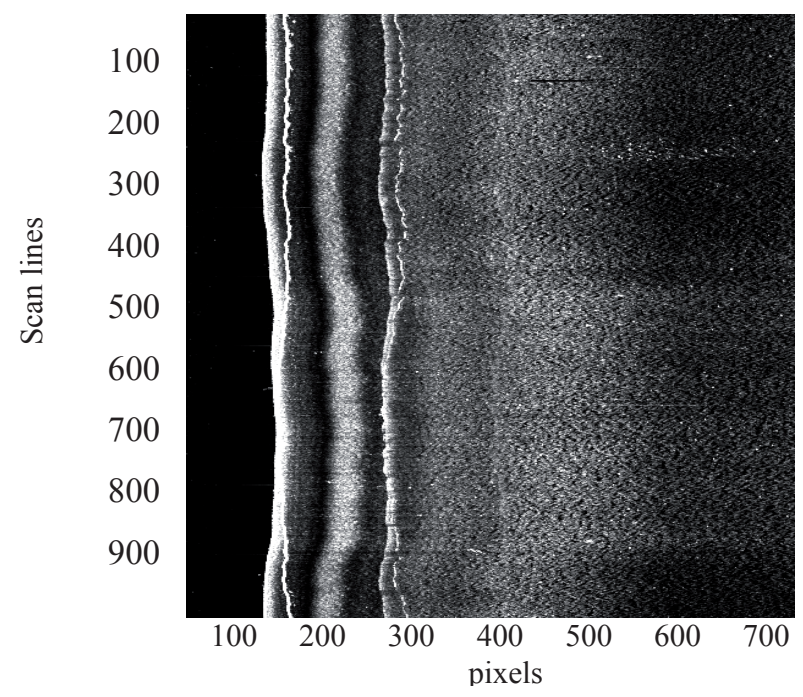

Fig. 2. Seabed side-scan image. the final image is generated from the echoes received from a single pulse of sound which is transmitted by the transducer arrays on both sides of the towfish. As the sensor is dragged through the water it repeatedly emits pulses and records the echoes, building the consecutive rows of the image.

The main strip in the center of the image is the area under the sonar and hence not actually part of the image and is not useful. Moving from the strip the rippled region corresponds to a sharp bottom return near the location of the sonar. The very bright white lines past the rippled region correspond to surface returns of the ocean surface.

The distance from the central line of the image can be equated with the sensor along the seabed. Range is related to the time taken between the transmission of the outgoing pulse and the reception of its echo. The brightness of the image at each pixel represents the amplitude of the echo that was received from the corresponding part of the scene.

Resultant images have resolution based on the setup and limitations of a particular side-scan sonar system. The resolution perpendicular to the direction of platform motion is related to the pulse length. Short pulses provide greater detail but they have less power than long pulses. Since they have less power, their range is reduced.

The resolution in the direction of motion, sometimes called the along track or azimuth resolution, is related to vessel speed, frequency of pings and, primarily, the sonar beam width. The beam can be narrowed by increasing the length of the sonar aperture, however, very long arrays become more cumbersome and difficult to maneuver. Spreading of the sonar beam causes resolution to diminish with range [30].

Since sound propagates so well under water, sonar technology is used widely for mapping the sea floor and ascertaining what is down there. This means that an abundance of sonar images is generated. Analyzing them is a highly skilled job. Due to various distortions, artifacts of noise and the nature of the image formation process itself, it can be difficult to read sonar images effectively.

Sonar images are often corrupted by noise during the process of their acquisition and transmission. The quality of the original images shown in Fig. 2 is non-exceptional. It is affected by various kinds of noise, including the different parameters that are presumed to be fixed (e.g., the range and fish height) during the process of their capturing. Image denoising is therefore necessary to remove the additive noise while retaining as much as possible the important image features.

Sonar images are inherently noisy due to various artifacts of acoustic imaging. To reduce the noise, an adaptive thresholding method is proposed in [31], preserving the structural features and textural information of sonar images. An assessment of the SQNR characteristics is rather informative prior to the application of many vision algorithms, including feature detection, matching and tracking in visual motion studies [32]. 
We used an approach based on statistical analysis over a large number of images of a stationary scene under relatively uniform imaging conditions. Ideally, averaging over all the overlapping pixels gives the ideal noisy-free image. For our data, however, we also have to account for the structured noise due to non-uniform illumination, variations in the imaging condition during data acquisition, and camera motion. These effects could be distinguished in the estimated distribution of the variances. Further processing by estimating the structured component as a constant multiplicative term within small local windows and subtracting output gives the error distribution due to sonar noise.

A preliminary experiment was carried out on sonar data and was aimed at demonstrating the estimation accuracy of the method when the data obey to the assumed models. Figure 3 reports modeled PDF of seabed image. The probability density function of pixel intensity is of interest because it describes the echo stability of a single object or portion of the bottom. A representative estimated PDF for this data set is shown in Fig. 3, a histogram of the image intensity values corresponding to a range at which a strong return is received from the bottom. The distribution of intensity values appears to be approximately Laplacian, described in the first part of the paper.

Usually, the gray-scale image is underexposed and blurry, due to underwater light limitations, seeing an example image in Fig. 2. The image is extremely dark; it lacks detail since the range of colors seems limited to low grey levels. We can verify this by looking at the image's histogram, illustrated in Fig. 3. In an image processing context, the histogram of an image normally refers to a histogram of the pixel intensity values. The image is scanned in a single pass and a running count of the number of pixels found at each intensity value is kept. It forms a tight, narrow peak cluster in the lower grey level region between the grey level intensity values of 0 to 50 , which means the whole image is represented almost entirely by dark pixels.

Due to the significance of correlation in DPCM system, in this paper we study the influence of correlation coefficients on $S Q N R$ for sonar images. Correlation coefficient is the measure of extent and direction of linear combination of two signals. If two signals are closely related with

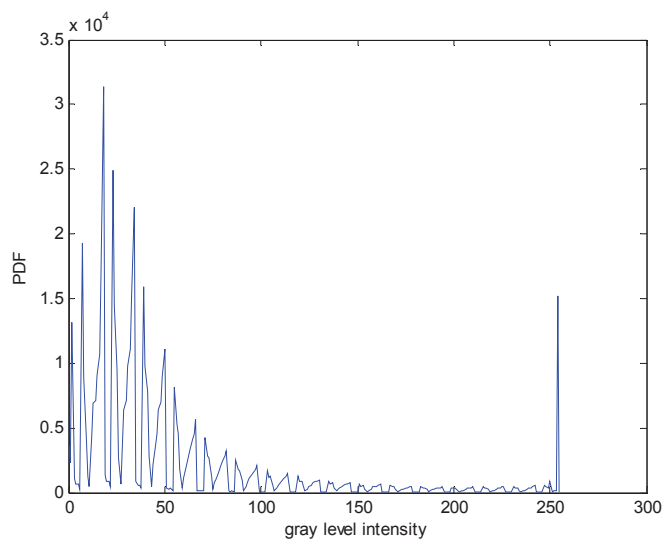

Fig. 3. Probability density function of a seabed image.

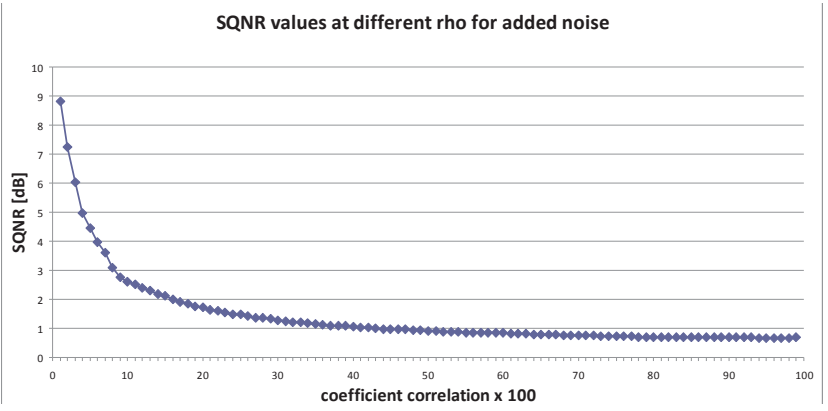

Fig. 4. $S Q N R$ values at different correlation coefficients for added noise.

stronger association, the correlation coefficient is close to value 1 . On the other hand, if the coefficient is close to 0 , two signals are not related and cannot predict each other. The prediction quality can be improved by adapting the correlation coefficient to the image content, e.g. edges or texture.

In the framework of this study, the $S Q N R$ is measured over all collected 511 sonar images. This measure compared the level of desired signal to the level of undesirable noise. $S Q N R$ is calculated between original full compression and noisy full compression to degree $\rho$ (rho). The resulting plot is the average $S Q N R$ value for each $\rho$ and it is shown in Fig. 4.

The case $\rho=1$ means it is the no noise case, but it has an $S Q N R$ value because of the rescaling of the histogram that was presented. We can see that $S Q N R$ decreases sharply when values for correlation coefficient approach the critical value 1. Danković and Perić, however, obtained sharp decrement of SQNR value when correlation coefficient equaled 0.8 for DPCM system [33]. D. Zogas et al. also measured dependence of $S Q N R$ on the correlation coefficient for wireless communications system [34]. They found as well that $S Q N R$ degrades as the correlation coefficient increases, but slightly compared to our system. On the other side, the algorithm presented in [35] can efficiently improve the $S Q N R$ of the sonar images.

\section{Conclusion}

This paper describes one model for information-theoretic assessment of digital images. It relies on robust noise estimation and entropy modeling in order to calculate information of the ideal noise-free signal starting from the observed digitized image. Thanks to predictive entropy modeling of information sources, we obtained the upper bound on the amount of information generated by an ideal noise-free process of sampling and digitization.

The theoretical estimation on upper bound on $S Q N R$ gain in DPCM entropy coding of images is obtained under the following constraints:

- The quantizer input has approximately the Laplacian probability density.

- The limiting degradation factor is quantizing noise. 
- The distortion criterion used is the squared-error criterion.

The full knowledge of the parametric noise model proves useful not only in applications requiring preliminary denoising, such as sonar imaging systems, but also in application of surveillance, where no denoising is performed.

The results of this paper can be applied to DPCM systems which don't change or adapt themselves to the statistics of the input signal. In adaptive systems, the quantizer levels may change in accordance with certain properties of the quantizer input signal. In this paper, we derived approximate expressions for the upper bound on the $S Q N R$ gain for image DPCM system with non-adaptive quantizer in closed form that depends only on the number of quantizing levels.

The following predictive image coding systems with white or correlated noise are observed concerning the $S Q N R$ gain: with entropy coding; with $\varepsilon$-entropy of the bit stream of the quantizer output as well as without entropy coding. The upper bounds on $S Q N R$ gain for $N=8$ quantizing levels are calculated to be 7.29 and $7.17 \mathrm{~dB}$ for systems with white and correlated noise, respectively, compared to the system without entropy coding. The gains predicted for entropy coding assume that the quantizer inputs are Laplacian. Moreover, models presented in this paper can be applied for any other signal with Laplacian distribution.

A better understanding of correlation in noise and target signals would provide the additional input to signal processing algorithms. Future work and further improvement of this model can be done using perceptual measures by taking the properties of human visual system. One possible extension of the system is to make it scalable, which means the coder is able to produce one bit stream that can be used to decode the source at different rates with different distortion levels. This attractive feature can be useful in image browsing, progressive transmission, and transcoding.

\section{Acknowledgments}

This study was supported by the Ministry of Education, Science and Technological Development of the Republic of Serbia $(32025,32048)$.

\section{References}

[1] HUCK, F. O., FALES, C. L., ALTER-GANTERBERG, R., PARK, S. K., RAHMAN Z. Information-theoretic assessment of sampled imaging systems. Journal Optical Engineering, 1999, vol. 38 , no. 5 , p. $742-762$. DOI: $10.1117 / 1.602264$

[2] AIAZZI, B., ALPARONE, L., BARDUCCI, A., BARONTI, S., PIPPI, I. Assessment of noise variance and information content of multi-/hyper-spectral imagery. ISPRS International Archives Photogrammetry and Remote Sensing, 1999, vol. 32, p. 164-174.

[3] AIAZZI, B., ALPARONE, L., BARDUCCI, A., BARONTI, S., PIPPI, I. Estimating noise and information of multispectral imagery. Journal Optical Engineering, 2002, vol. 41, no. 3, p. 656-668. DOI: $10.1117 / 1.1447547$

[4] BLAHUT, R. E. Principles of Information Theory. Reading (MA): Addison-Wesley, 1987.

[5] SHANNON, C. E., WEAVER, W. The Mathematical Theory of Communication. Urbana (IL): University of Illinois Press, 1949.

[6] PARK, S. K., RAHMAN, Z. Fidelity analysis of sampled imaging systems. Journal Optical Engineering, 1999, vol. 38, no. 5, p. $786-800$. DOI: $10.1117 / 1.602047$

[7] RAGESH, N. K., ANIL, A. R., RAJESH, R. Digital image denoising in medical ultrasound images: a survey. In Proceedings of the ICGST AIML-11 Conference. Dubai (UAE), 2011, p. 67-73.

[8] FAN, G., XIA, X. G. Image denoising using a local contextual hidden Markov model in the wavelet domain. IEEE Signal Processing Letters, 2001, vol. 8, no. 5, p. 125-128. DOI: 10.1109/97.917691

[9] BERGER, T. Rate-Distortion Theory. John Wiley \& Sons, 1971.

[10] O’NEAL, J. B. Predictive quantizing systems/DPCM for the transmission of television signals. Bell Systems Technology Journal, 1966, vol. 45, p. 689-721.

[11] KIVANC, M., KOZINTSEV, M., RAMCHANDRAN, I., MOULIN, P. Low-complexity image denoising based on statistical modeling of wavelet coefficients. IEEE Signal Processing Letters, 1999, vol. 6, no. 12, p. 300-303. DOI: 10.1109/97.803428

[12] AIAZZI, B., ALPARONE, L., BARONTI, S., LOTTI, F., SANTURI, L. A model for estimating noise and information of digital signals: an evaluation on medical images. In Proceedings of the International TICSP Workshop on Spectral methods and Multirate Signal Processing SMMSP 2003. Barcelona (Spain), September 2003, p. 83-88.

[13] KOLMOGOROV, A. N. Theory of transmission of information. Annales Mathematical Society Translations, 1963, vol. 33, no. 2, p. 291-321.

[14] JERZMAN, B., KICINSKI, W. Kernel estimation of probability density function: properties and parameters optimization. Metrology and Measurement Systems, 2009, vol. XVI, no. 1, p. 85-105.

[15] JAYANT, N. S., NOLL, P. Digital Coding of Waveforms: Principles and Applications to Speech and Video. Englewood Cliffs (NJ): Prentice-Hall, 1984.

[16] ISAR, A., MOGA, S., ISAR, D. Denoising sonar images using a bishrink filter with reduced sensitivity. Rev. Roum. Sci. Techn. Electrotechn. Et Energ., Bucharest, 2011, vol. 55, no. 2, p. 181 to 190.

[17] ISAR, A., FIROIU, I., NAFORNITA, C., MOGA, S. Sonar images denoising. Sonar Systems, edited by Nikolai Kolev, InTech, 2011. ISBN: 978-953-307-345-3

[18] SHI, HONG, CHUNHUI ZHAO., ZHENGYAN SHEN, GUANGYU LIU. Sonar image fusion denoising method based on multiple morphological wavelet packets. Advanced Electrical and Electronics Engineering, Springer Berlin Heidelberg, 2011, vol. 2, p. 689-696. ISBN: 978-3-642-19711-6

[19] BOJKOVIĆ, Z. Upper bound on signal/quantising-noise ratio for television D.P.C.M. systems. Electronics Letters, 1975, vol. 11, no. 1, p. 29-30. DOI: 10.1049/el:19750021

[20] HABIBI, A. Hybrid coding of pictorial data. IEEE Transactions on Communications, 1974, vol. 22, no. 5, p. 614-624. DOI: 10.1109/TCOM.1974.1092258 
[21] WOOD, R. C. On optimum quantization. IEEE Transactions on Information Theory, 1969, vol. IT-15, no. 2, p. 248-252. DOI: 10.1109/TIT.1969.1054285

[22] PANTER, P. F., DITE, W. Quantization distortion in pulse-count modulation with nonuniform spacing of levels. Proceedings IRE, 1951, vol. 39, p. 44-48.

[23] NITADORI, K. Statistical analysis of DPCM. Journal Electron. Communications Japan, vol. 48, 1965.

[24] WEINBERGER, M. J., RISSANEN, J. J., ARPS, R. B. Applications of universal context modeling to lossless compression of gray-scale images. IEEE Transactions on Image Processing, 1996, vol. 5, no. 4, p. 575-586. DOI: 10.1109/83.491334

[25] BENNETT, W. R. Spectra of quantized signals. Bell Systems Technology Journal, 1948, vol. 27, no. 3, p. 446-472.

[26] MilovanOViĆ, D., BOJKOVIĆ, Z., SAMČOVIĆ, A. Upper bound on SNR gain in wavelet transform predictive-entropy image coding. Journal of Circuits, Systems and Computers, 1998, vol. 8 , no. 2, p. 267-272. DOI: 10.1142/S0218126698000109

[27] SAMČOVIĆ, A., BOJKOVIĆ, Z. Redundancy reduction in entropy coding of wavelet images. In Proceeding of the Symposium on Electronics and Telecommunications Etc.'98. Timisoara (Romania), September 1998, vol. II, p. 176-180.

[28] MILOVANOVIĆ, D., BOJKOVIĆ, Z., SAMČOVIĆ, A., RELJIN, B. Upper bound on redundancy reduction in predictive-entropy subband image coding. China Journal of Image and Graphics, 1997, vol. 2, no. 4, p. 239-249.

[29] CUSCHIERI, J. M., LEBLANC, L. R., PAZOL, B., PHAM, H. Forward look Sonars for AUV's, 2000.

[30] CHANUSSOT, J., MAUSSANG, F., HETET, A. Scalar image processing filters for speckle reduction on synthetic aperture sonar images. In Proceedings of the Oceans '02 MST/IEEE. 2002, vol. 4, p. 2294-2301. DOI: 10.1109/OCEANS.2002.1191987

[31] MODAlavalasa, N., RAO G. S., SATYA PRASAD, K. A novel approach for segmentation of sector scan sonar images using adaptive thresholding. International Journal of Information Sciences and Techniques, July 2012, vol. 2, no. 4, p. 113-119. DOI: $10.5121 /$ ijist.2012.2411
[32] NEGAHDARIPOUR, S., FIROOZFAM, P., SABZMEYDANI, P. On processing and registration of forward-scan acoustic video imagery. In Proceedings of the $2^{\text {nd }}$ Canadian Conference on Computer and Robot Vision CRV'05. 9-11 May 2005, p. 452-459. DOI: 10.1109/CRV.2005.57

[33] DANKOVIĆ, N., PERIĆ, Z. Probability of stability estimation of DPCM system with the first order predictor. Facta Universitatis, Series: Automatic Control and Robotics, 2013, vol. 12, no. 2, p. 131-138. ISSN: 1820-6417

[34] ZOGAS, D., KARAGIANNIDIS, G. K., KOTSOPOUlOS, S. A. On the average output SNR in selection combining with three correlated branches over Nakagami-m fading channels. IEEE Transactions on Wireless Communications, 2004, vol. 3, no. 1, p. 25-28. DOI: 10.1109/TWC.2003.821219

[35] LUO, J., LIU, H., HUANG, C., GU, J., XIE, S., LI, H. Denoising and tracking of sonar video imagery for underwater security monitoring systems. In Proceedings of the IEEE International Conference on Robotics and Biomimetics ROBIO 2013. Shenzhen (China), 12-14 December 2013, p. 2203-2208. DOI: 10.1109/ROBIO.2013.6739796

[36] WONG, L. S., ALLEN, G., EVANS, B. Sonar data compression using non-uniform quantization and noise shaping. In Proceedings of the Asilomar Conference on Signals, Systems, and Computers. Pacific Grove (CA, USA), 2-5 November 2014, p. 1895-1899. DOI: 10.1109/ACSSC.2014.7094798

\section{About the Author ...}

Andreja SAMČOVIĆ received his $\mathrm{PhD}$ from the University of Belgrade in 2005. His research interests include multimedia communications, image and video coding and compression. He is involved in the COST IC 1106 and 1206 actions. He is an associate professor at the University of Belgrade for Information and Communication Technologies. 